\title{
The Role of Pretraining on Skilled Forelimb Use in an Animal Model of Huntington's Disease
}

\author{
R. A. Fricker-Gates, R. Smith, J. Muhith, and S. B. Dunnett \\ Brain Repair Group, School of Biosciences, Cardiff University, UK
}

\begin{abstract}
After a unilateral striatal lesion, animals have generally been seen to have a bilateral impairment in paw reaching, with the contralateral paw being more affected. However, most studies to date have not used a pretraining paradigm to assess maximal capacity for paw reaching, to compare with any lesion-induced loss. This study compared animals that were pretrained with naive animals in their ability to paw reach after a striatal lesion, to address the role of the striatum in either acquisition or execution of this motor task. All lesioned animals showed a significant decrease in reaching ability with their contralateral paw compared with the ipsilateral paw. Pretrained lesioned animals showed a clear lesion deficit with the contralateral paw immediately after lesion, and no impairment whatsoever with the ipsilateral paw. Naive lesioned animals showed delayed acquisition of the task with both paws, possibly due to postural deficits, and a lasting deficit on the contralateral side. The variability of performance between animals was higher in the naive lesioned group. These results suggest that animals should be pretrained on the staircase task prior to lesion to enable maximum sensitivity in detecting both loss and recovery of function of skilled forelimb use.
\end{abstract}

Key words: Striatal lesion; Skilled forelimb use; Quinolinic acid; Motor functions

\section{INTRODUCTION}

Degeneration of the medium spiny projection neurons within the dorsolateral striatum causes a marked impairment in skilled motor functions $(5,20)$. Thus, in the excitotoxic lesion model of Huntington's disease (HD), loss of striatal projection neurons can be measured using tests of skilled forelimb use such as the "staircase task" $(1,16)$. In this task, animals are trained to reach for food pellets placed in wells of a staircase descending either side of a central platform. To successfully retrieve a pellet the animal must execute a reaching movement, grasp a pellet, and return it to the mouth; hence, this task requires complex motor function. Skilled forelimb movements such as these have been shown to require the integrity of striatal projection neurons, the nigrostriatal dopaminergic system, and sensorimotor cortex (23).

In the studies to date assessing the effects of striatal neuron depletion on skilled paw reaching, the staircase task has been used in a number of forms, and experimental designs have varied widely. For instance, Nakao and colleagues used a staircase with four large wells each holding 10 pellets $(17,18)$, while our groups have preferred a longer staircase with wells holding 2-3 pellets $(1,6,22)$. In addition, only a few studies have included an unoperated control group $(5,10,11,17)$, and there has been a great deal of variation in the number of test days before animals have been recorded as reaching asymptotic levels of success $(6,15,18)$. Strikingly, prelesion training of animals in the staircase task has seldom been tried in studies of skilled forelimb use $(12,15)$.

A unilateral excitotoxic lesion to the dorsolateral striatum leads to impairment in skilled forelimb use with the contralateral paw, which remains stable over time $(6,15,18)$. In addition, many studies report a more modest impairment with the ipsilateral paw, although this has not always been observed (17). It is unclear whether these differences in outcome reflect the experimental design, or perhaps the degree or site of striatal neuron loss $(5,17,20)$.

With the integrity of cortico-striatal circuitry and attenuating dopaminergic inputs to the striatum all playing a role in skilled forelimb use, we hypothesized that the integrity of this motor loop could play a role in execution and/or learning of the reaching task, or could affect postural stability (nonspecific role), as suggested previously (15). Therefore, we designed a controlled lesion study, to compare animals that had been pretrained to maximal success levels on the staircase task with naive animals, in an attempt to tease out the factors underlying paw reaching ability after a quinolinic acid lesion to the dorsolateral striatum.

Address correspondence to Rosemary A. Gates, School of Biosciences, Cardiff University, Museum Avenue, P.O. Box 911, Cardiff CF10 3US, Wales, UK. Tel: 0292087 5188; Fax: 0292087 6749; E-mail: gatesra@cf.ac.uk 


\section{MATERIALS AND METHODS}

Thirty-two adult female Sprague-Dawley rats were used in this study. All animals were housed in groups of four animals per cage under a natural light-dark cycle. Food and water were available ad lib, other than during the training and testing of the staircase task, during which animals were maintained on a restricted diet each day, given directly after the training/test session, to maintain $95 \%$ normal body weight. Rats were allocated randomly into two groups: 16 received training on the staircase task prior to lesion, the remainder received no training, but were also maintained on the same restricted diet. The time course for the experiment meant that pretrained animals received 28 sessions of training followed by a forced choice test, following which animals were transferred to an ad lib diet for 10 days both pre- and postsurgery. The prelesion rotation to amphetamine was conducted 5 days prior to lesioning. Following the lesion all animals completed 28 sessions of paw reaching followed by the forced choice test, after which all were transferred to an ad lib diet, 1 week prior to the postlesion amphetamine rotation test.

\section{The "Staircase" Task}

In this test of skilled forelimb use, the animal has to reach for 45-mg Noyes sugar pellets placed in wells made in each step of a descending staircase, on either side of a raised central platform. The apparatus is designed so that only the paw on the same side as each staircase can be used, thus providing a measure of independent forelimb use. To retrieve a pellet, the animal must make a coordinated reach, grasp the pellet, and deliver it to the mouth with the paw. Success is scored as the number of successful retrievals in a 15-min session. Three pellets placed in each well gave a maximal score of 21 pellets per side. After two sessions of habituation to the box, animals received 28 daily sessions of 15 min. After training was complete, each animal underwent a "forced choice" test of two separate 10-min sessions where only one of the two randomized sides was baited with pellets in any session, alternating to the other side in the second session, to test the maximal capacity for reaching when constrained to use only one paw at a time.

Pretrained rats were then divided to two groups, matched on paw reaching ability with both paws $(n=8$ per group). Group A received a striatal lesion (see below) while group B remained as intact pretrained controls. Half the naive animals $(n=8)$, group $\mathrm{C}$, also received a striatal lesion. Group D $(n=8)$ remained as intact naive controls. Four of the 16 pretrained animals showed some side preference (one to the right side and the remainder to the left). All other animals showed no preference for a particular paw. These four animals were assigned to the two groups such that analysis of variance of group scores revealed no significant differences between left or right paws. Based on this, all animals were lesioned on the right side, regardless of whether they showed any particular side bias, and such that the choice of ipsilateral (lesioned) side was not biased in favor of the three animals whose preference was to the left side, making the lesion effect in these animals more robust. Ten days after the lesion all naive animals (groups $\mathrm{C}$ and D) received two sessions of habituation in the boxes. All groups were then tested for paw reaching ability over a further 28 15-min sessions, followed by a forced choice test.

Data for paw reaching were grouped into blocks of four trials and analyzed for numbers of pellets consumed by two-factor analysis of variance (ANOVA), with the Groups as a single between-subjects factor and Sides (contralateral vs. ipsilateral to the lesion) as the withinsubject factor. ANOVA was carried out using Genstat 5.3 (Numerical Algorithms Group, AFRC, Rothampstead, UK). Newman-Keuls tests were used for post hoc comparisons between groups.

\section{Rotation}

Amphetamine-induced rotation was evaluated in a bank of eight rotometer bowls modeled after the design of Ungerstedt and Arbuthnott (21). The number of rotations was calculated over a 90-min period, after an IP injection of $5 \mathrm{mg} / \mathrm{kg}$ D-amphetamine (Sigma). One rotation test was undertaken 5 days prior to lesioning, and the second at 9 weeks postlesion after completion of the paw reaching trials. Data were analyzed by two-factor (Group $\times$ Test) ANOVA.

\section{Lesion Surgery}

All animals in the two lesion groups received a unilateral quinolinic acid lesion to the dorsolateral striatum. Animals were anesthetized with $1-2 \%$ isofluorane, vaporized in a 2:1 mixture of oxygen and nitrous oxide, and mounted in a Kopf 900 stereotaxic frame with the incisor bar set at $-2.3 \mathrm{~mm}$ below the interaural line. Quinolinic acid dissolved in phosphate-buffered saline (PBS) $(0.09 \mathrm{M}, \mathrm{pH} 7.4)$ was infused at a rate of $0.25 \mu \mathrm{l} /$ min over 3 min via a 30 -gauge stainless steel cannula. Coordinates used were: $\mathrm{A}=0.6, \mathrm{~L}=-3.1, \mathrm{~V}=-4.5$. The cannula was left in place for a further 3 min before retraction and the wound sutured. Animals received 0.15 $\mathrm{ml}$ diazepam IM just prior to removal from the frame. Nonlesioned animals received no surgery, but remained as unoperated controls.

\section{Histology}

At the completion of all behavioral experiments, animals were anesthetized with $1 \mathrm{ml}$ sodium pentobarbitone 
IP and perfused transcardially with $200 \mathrm{ml} 0.1 \mathrm{M}$ PBS (pH 7.4) followed by $300 \mathrm{ml} 4 \%$ paraformaldehyde in PBS. The brains were removed and placed in fixative for $24 \mathrm{~h}$. They were then transferred to a $25 \%$ sucrose solution in PBS for sectioning. Sections were cut on a freezing microtome at a thickness of $40 \mu \mathrm{m}$.

A 1:6 series of sections underwent immunohistochemistry for the dopamine adenosine cAMP receptorrelated phosphoprotein of $32 \mathrm{kDa}$ (DARPP-32) to visualize the medium spiny striatal projection neurons. Endogenous peroxidase activity was quenched using a 5 -min incubation with a solution containing $10 \%$ methanol and $10 \%$ hydrogen peroxide $\left(\mathrm{H}_{2} \mathrm{O}_{2}\right)$. Following three rinses, the free-floating sections were incubated for $1 \mathrm{~h}$ in a blocking solution containing 3\% normal horse serum and $0.2 \%$ Triton in Tris-buffered saline (TBS). The DARPP-32 antibody (a kind gift from Prof. Hugh C. Hemmings) was used at 1:20,000 in blocking solution, and the sections incubated for $36 \mathrm{~h}$ at $4^{\circ} \mathrm{C}$. Horse antimouse secondary antibody (DAKO) was used at a concentration of 1:200, followed by avidin-biotin complex (DAKO) also at 1:200. The DARPP-32 was visualized using the 3,3'-diaminobenzidine (DAB) chromogen.

The extent of the striatal lesion was quantified by measurement of volume loss on the lesion side compared with the intact contralateral rostral dorsal striatum. Cross section areas were measured including regions bordered by the corpus callosum dorsally, the external capsule laterally, and the lateral ventricle medially, and by a line angled $23^{\circ}$ to the horizontal and tangential to the dorsal margin of the anterior commissure. A series of one in eight coronal sections was used for area measurements, from the anterior striatum until the point of decussation of fibers of the anterior commissure (i.e., 9-11 sections per animal). Areas were recorded at low magnification using a WILD macroscope (WILD Heerburg, Switzerland) and SCION image analysis software (SCION Corporation, Frederick, MD). Volumes were estimated by integrating the cross-sectional areas by the interval between sections $(320 \mu \mathrm{m})$. The two lesion groups were compared using a two-tailed $t$-test.

\section{RESULTS}

\section{Histology}

The extent of the striatal lesions is illustrated in Figure 1. After a quinolinic acid injection there was a total loss of DARPP-32-positive medium spiny neurons throughout the central and lateral regions of the rostral striatum. As has been previously observed with this excitotoxin, the extreme medial striatal and ventral nucleus accumbens regions were spared from neuronal damage. The zone of neuronal degeneration could be easily delineated, allowing the extent of loss of striatal volume to be estimated. Estimates of residual striatal volume on the lesion side were compared with striatal volumes of the contralateral intact side for each animal to calculate the percentage of striatal volume loss for each animal. The percentage of volume loss in the rostral striatum did not differ when comparing the two lesion groups: pretrained, $67.1 \pm 1.9 \%$ vs. naive, $71.7 \pm 2.6 \%, t(13)=$ 1.47 , n.s.

\section{Rotation}

After a unilateral quinolinic acid lesion, both lesion groups showed a rotational bias ipsilateral to the lesion [i.e., turning towards the right (lesioned) side] (Fig. 2). No side bias was seen in either of the two control nonlesioned groups. ANOVA of seven amphetamine-induced rotations showed a significant effect of the lesion [Rotation $\times$ Lesion interaction, $F(1,28)=14.74, p=0.001]$. There was no significant difference in rotational asymmetry between the pretrained or naive lesion groups, $F(1,28)=0.24$, n.s.

\section{The Staircase Task}

During the 28-session pretraining period both pretrained groups acquired the paw reaching task and reached asymptotic levels of pellets reached with both paws (lesioned group $=12.5 \pm 0.8$ vs. $13.3 \pm 0.6$ with the ipsilateral and contralateral paws, respectively; nonlesioned group $=12.5 \pm 1.3$ vs. $13.9 \pm 1.0$ ) (Fig. 3A, B)

At the beginning of the second testing period, the nonlesioned pretrained group retained the ability to reach with both paws at the same level as seen at the end of the pretraining period, reaching $13.9 \pm 1.2$ pellets with the ipsilateral and $14.5 \pm 1.2$ pellets with the contralateral paw (Fig. 3A). The pretrained lesioned group retained the ability to reach with their paw ipsilateral to the lesion, but showed a significant deficit with the paw contralateral to the lesion, reaching $14.5 \pm 0.4$ pellets with the ipsilateral and $11.8 \pm 0.6$ pellets with the contralateral paw, respectively, in the final block of testing (Fig. 3B) [Side $\times$ Test: $F(1,14)=7.92, p=0.01]$. This deficit was apparent from the first day of retesting and the success with the contralateral paw continued to be significantly different to the ipsilateral paw throughout the 28-session testing period.

The naive nonlesioned rats learned the paw reaching task equally well with both paws and at a similar rate to the pretrained animals (Fig. 3C). The naive lesioned group showed a slower rate of acquisition of the task with both paws when compared with the naive nonlesioned animals (Fig. 3C). Post hoc Newman-Keuls tests revealed that at the final block of testing, the naive lesioned group had reached a similar level of success with their ipsilateral paw as all other groups $(12.9 \pm 1.3$ pellets). However, the naive lesioned group showed a significant deficit with their contralateral paw when com- 




Figure 1. Coronal sections through the center of the striatal lesion in a pretrained (A) and nonpretrained animal (B). Images show DARPP-32 labeling of striatal medium spiny neurons at $2 \times$ magnification (see Materials and Methods for immunohistochemistry details). Note the loss of DARPP-32 immunoreactivity in a discrete region of the dorsolateral striatum, which does not differ in size between animals. Scale bar: $1 \mathrm{~mm}$.



Figure 2. Rotation in response to $5 \mathrm{mg} / \mathrm{kg}$ D-amphetamine, measured as net ipsilateral (right) turns, both prelesion and at 4 weeks postlesion. Each bar represents mean turns per group per 90min session $( \pm \mathrm{SEM})$. Both lesioned groups show a similar rate of ipsilateral turning postlesion, which is significantly greater than both intact groups. PT, pretrained. 


\section{A. Pretrained animals - Intact}

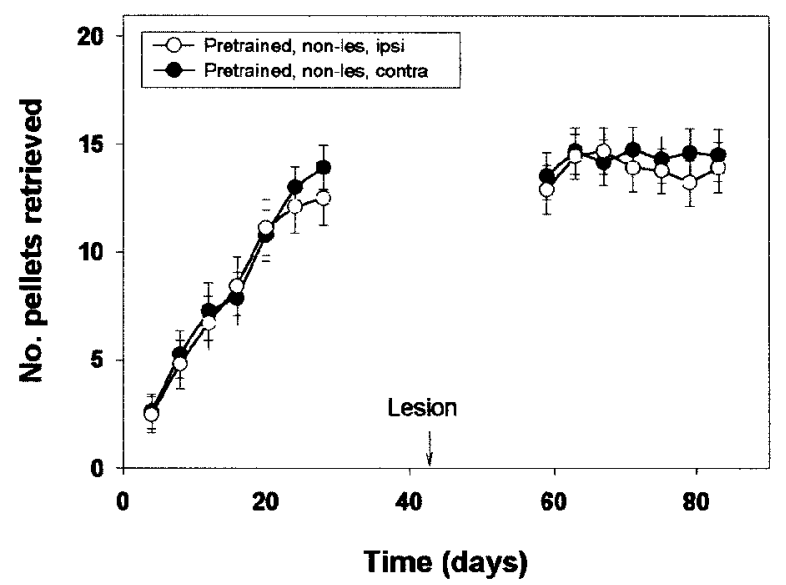

C. Naive animals

Lesion vs. Intact



B. Pretrained animals - QUIN lesion

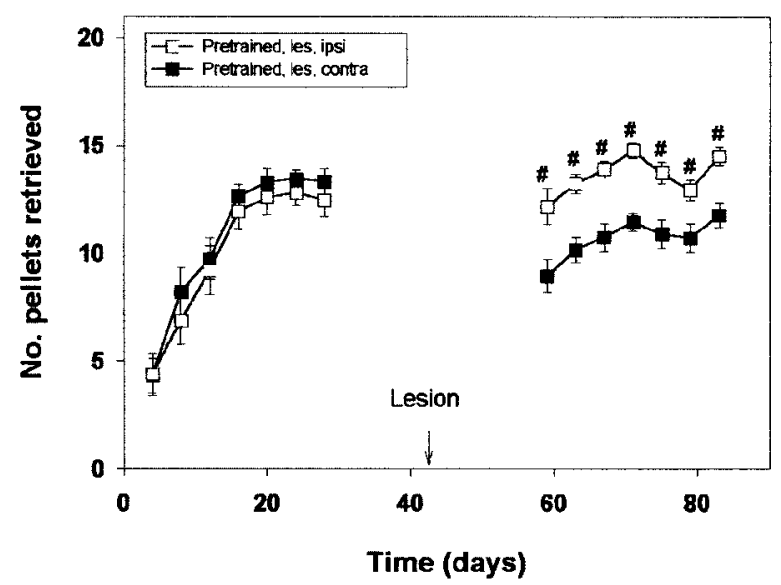

\section{Lesioned animals \\ Pretrained vs. Naive}

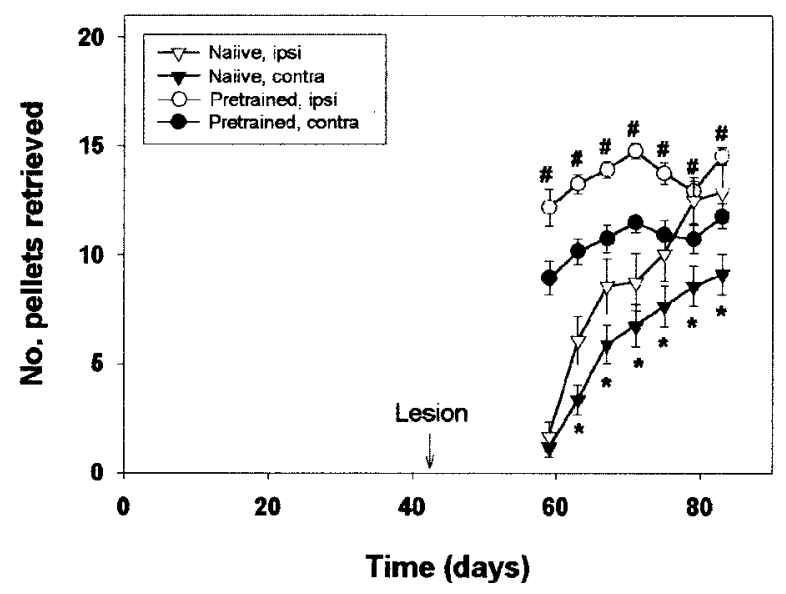

Figure 3. Skilled paw reaching in the staircase test. Each plot represents the mean number of pellets retrieved per group $( \pm$ SEM) per block of four consecutive trials. (A) Pretrained nonlesioned animals. (B) Pretrained lesioned group. (C) Comparison of both naive groups, nonlesioned and lesioned. (D) Comparison of both lesioned groups, pretrained and naive. \#Significant difference between ipsi- and contralateral paws within the pretrained lesioned group. *Significant difference between ipsi- and contralateral paws within the naive lesioned group.

pared with the ipsilateral paw, only reaching $9.1 \pm 0.9$ pellets [Side $\times$ Lesion: $F(1,14)=7.32, p=0.02]$ (Fig. 3C). This contra-ipsi deficit was similar in size to that of the pretrained animals, and apparent from the second block of testing (Fig. 3D).

The difference between the number of pellets retrieved with the ipsilateral and contralateral paws was compared between all groups in the final 4-day block of the 28-session postlesion period. ANOVA revealed a highly significant effect of the lesion, $F(1,28)=20.27$, $p<0.001$ (Fig. 4). The pretrained lesioned group reached $14.5 \pm 0.4$ pellets with the ipsilateral and $11.8 \pm 0.6$ pellets with the contralateral paw, while the naive group reached $12.9 \pm 1.3$ pellets with the ipsilateral and $9.1 \pm$ 0.9 pellets with the contralateral paw. However, the magnitude of the ipsi-contralateral difference was not significantly different between either the two lesioned groups or the two nonlesioned groups, regardless of whether they had received pretraining or not [Training $\times$ Lesion: $F(1,28)=0.11$, n.s.]. Inspection of Figure 


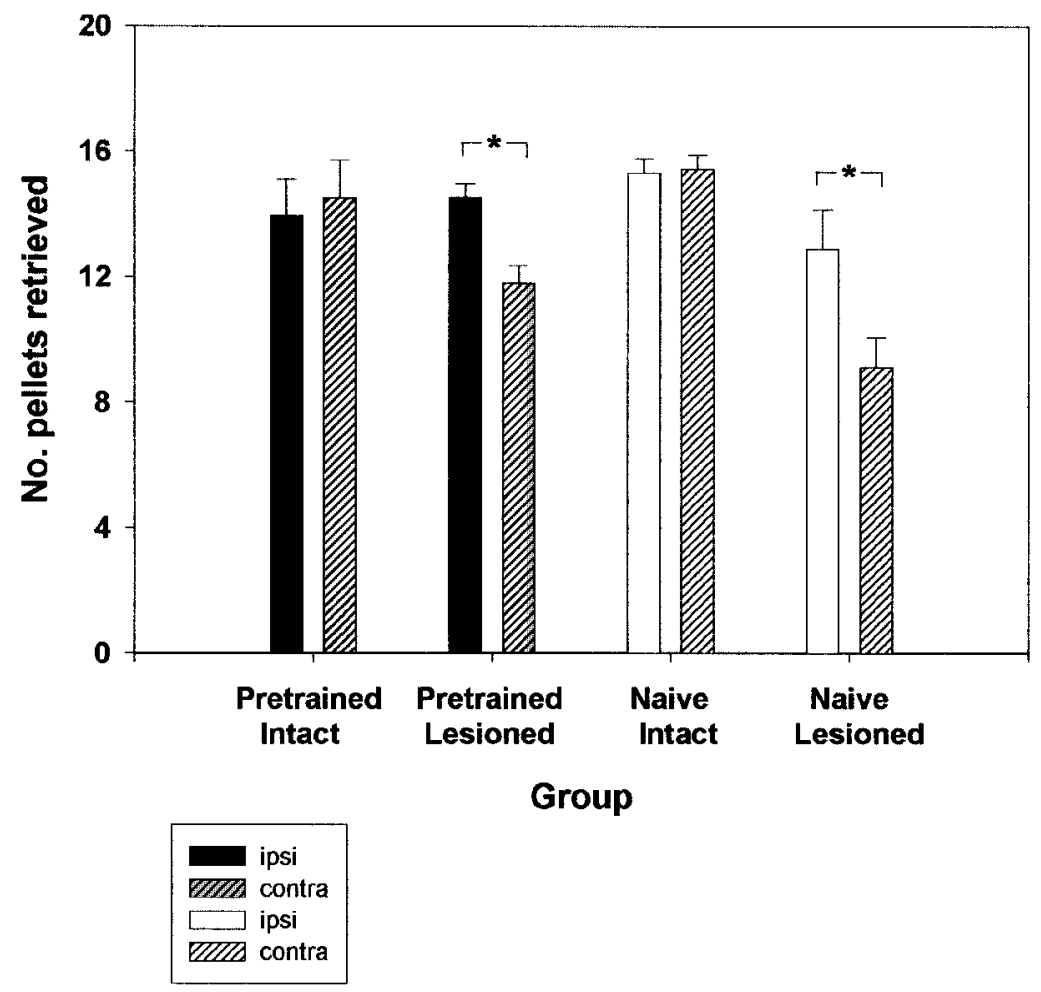

Figure 4. Comparison of all groups over the final four sessions of paw reaching. Bars indicate mean number of pellets retrieved per group $( \pm$ SEM $)$. *Significant difference between the ipsi- and contralateral paws.

4 indicates that the variance of reaching performance among lesioned rats appears greater among the naive than pretrained rats, on both sides. Variance ratio tests indicated that this was highly significant on the intact (ipsilateral) paw, $F(7,7)=35.8, p<0.001$, and approached but did not reach significance on the impaired (contralateral) side, $F(7,7)=3.28, p<0.1$.

Results of the forced choice tests reflected a similar pattern, although ANOVA tests did not reveal a significant difference between groups or sides. In both lesion groups there was a deficit in number of pellets retrieved with the contralateral versus ipsilateral paw $(11.0 \pm 1.8$ vs. $13.6 \pm 0.8$ in the pretrained group, $9.5 \pm 1.7$ vs. $14.0 \pm$ 2.4 in the naive group). The nonlesioned groups maintained a similar ability to reach with either paw during the forced choice test $(14.6 \pm 2.3$ pellets were retrieved with the contralateral vs. $13.6 \pm 2.2$ with the ipsilateral paw in the pretrained group, and $15.1 \pm 0.9$ vs. $15.4 \pm$ 0.8 in the naive group). Lack of a significant side bias in the lesioned groups suggests that there is increased motivation to use the impaired paw when the reward is restricted to that side, and the animals persevere with reaching beyond the level seen when they have addi- tional and more easily accessible pellets on the ipsilateral side.

Therefore, in summary, both pretrained and naive animals showed a significant deficit with their contralateral paw following the quinolinic acid lesion. This deficit did not change depending on whether the animals had been pretrained or not. However, pretraining provided a stable baseline from which a lesion deficit in the contralateral paw was observed immediately when the postlesion testing commenced. In addition, the pretrained lesioned group also showed less variability around the group mean than the naive lesion group, in terms of pellets collected with either the ipsi- or contralateral paws (Fig. 3D). The naive lesioned group also showed a slower rate of acquisition of the task with both ipsi- and contralateral paws.

\section{DISCUSSION}

In this study we show that pretraining does not affect the outcome of a quinolinic acid lesion on the ability of rats to reach with either their ipsilateral or contralateral paw. However, pretrained rats showed lower variability of paw reaching success subsequent to the lesion, and 
the lesion effect on the contralateral paw was both immediate and sustained over time. In addition, naive lesioned animals showed a slower rate of acquisition of the task, with both ipsi- and contralateral paws, compared with naive intact controls.

The loss of skilled forelimb use with the contralateral paw after a unilateral excitotoxic lesion to the striatum has been well documented $(6,12,15,18)$. Here we show a marked impairment with the contralateral paw in both lesion groups when comparing ipsi- vs. contralateral reaching ability (i.e., the loss of medium spiny striatal projection neurons caused impairment of execution of paw reaching with the contralateral paw, independent of the rats' prior training in the staircase test). The loss of medium spiny striatal projection neurons did not affect the animals' ability to acquire the paw reaching task and after 28 days naive animals performed as well as pretrained animals with both paws. Therefore, bilateral integrity of the cortico-striatopallidal loop is not essential for acquisition of this motor task, and it appears from these data that the lesioned animals have a purely motor deficit. It is known that information is distributed bilaterally in the input pathways to the striatum $(4,24)$ and that output pathways from the basal ganglia are not completely lateralized $(8,10)$. Therefore, it remains possible that the striatum, as part of the cortico-striato-pallidal loop, plays a role in acquisition of skilled forelimb use, but that this would only be exposed using a bilateral lesion paradigm.

Pretraining, however, did proffer an advantage to the analysis of the behavioral deficit. The effects of the lesion on skilled forelimb use in pretrained animals were immediate, stable throughout the postlesion period, and showed little variability around the mean. In contrast, naive animals showed a greater variability in paw reaching success and, as a group, had not reached maximal scores by the end of 28 testing sessions. In addition, there was a lag in reaching ability of naive lesioned animals compared with naive intact controls over the first 20 days of testing. This lag may have been due to a dysfunction in postural adjustment in the paw used for balance, leading to impaired control of reaching with the other paw, particularly at longer distances (23). This factor has been suggested to play a role in the skilled reaching deficits of rats with unilateral dopamine depletions (14). Indeed, the pretrained lesioned animals showed a slight increase in reaching ability with both paws over the 28-day postlesion test period, consistent with increased postural control.

The quinolinic acid dose and delivery method used in this study created a stable but moderate lesion, with extensive loss of DARPP-32-positive projection neurons, but little tissue collapse or ventricular enlargement, and no extraneous damage in either the lateral or dorsal cortex. Equally, lesioned rats showed only moderate rotational bias (approximately 3 turns per minute) and moderate (20-30\%) deficits in reaching with the contralateral paw. Therefore, it cannot be excluded that the effects of pretraining in animals with larger lesions may show different and possibly more pronounced effects, particularly with reaching ability of the ipsilateral paw, which has been shown to be affected in some lesion paradigms $(6,15)$, but was not significantly impaired in this study. A more moderate but targeted lesion paradigm was chosen specifically to restrict toxin flow to the dorsolateral striatum, to be able to dissociate any behavioral deficits observed from extraneous factors such as damage to cortical or pallidal neurons involved in the motor circuitry used in skilled motor functions.

From these data several conclusions can be drawn. The stable and immediate deficit shown by pretrained animals allows stronger statistical power of analysis, with only limited testing immediately postlesion and after future interventions, whereas naive animals require extensive postlesion testing until asymptote levels of success are obtained. Even then the deficit turns out to be less significant, not because there is any difference in the magnitude of deficit between naive and pretrained animals, but because of the greater variability in the performance of the latter. The greater variance is further exacerbated by the fact that in the absence of pretraining, the groups cannot be matched or balanced on the basis of prelesion performance, which may be an important consideration when exploring therapeutic interventions such as neuronal protection $(3,7,13,19)$ or cell replacement therapies (2), when subtle changes in performance may result. In addition, the lack of pretraining in many studies, and the variation in length of testing postlesion/transplant, may explain some of the discrepancies in paw reaching deficits observed between different research groups $(6,15,18)$.

In conclusion, pretraining of animals on the staircase task provides a stable and less variable baseline, and is recommended for all future studies, to enhance both the observation of and statistical robustness of behavioral deficits in rat models of neurodegeneration.

ACKNOWLEDGMENTS: We wish to thank Professor Hugh C. Hemmings for the generous gift of the DARPP-32 antibody. R.A.F.-G. and R.S. are supported by the Wellcome Trust. This work was supported by the Medical Research Council.

\section{REFERENCES}

1. Abrous, D. N.; Dunnett, S. B. Skilled paw reaching in rats: The staircase test. Neurosci. Prot. 3:1-11; 1994.

2. Dunnett, S. B.; Nathwani, F.; Björklund, A. The integration and function of striatal grafts. In: Björklund, A.; Dunnett, S. B., eds. Functional neural transplantation II: 
Novel cell therapies for CNS disorders. New York: Elsevier Science; 2000:345-380.

3. Emerich, D. F.; Lindner, M. D.; Winn, S. R.; Chen, E. Y.; Frydel, B. R.; Kordower, J. H. Implants of encapsulated human CNTF-producing fibroblasts prevent behavioral deficits and striatal degeneration in a rodent model of Huntington's disease. J. Neurosci. 16:5168-5181; 1996.

4. Fisher, R. S.; Shiota, C.; Levine, M. S.; Hull, C. D.; Buchwald, N. A. Subcortical crossed axonal projections to the caudate nucleus of the cat: A double-labelling study. Neurosci. Lett. 51:25-30; 1984.

5. Fricker, R. A.; Annett, L. E.; Torres, E. M.; Dunnett, S. B. The locus of a striatal ibotenic acid lesion affects the direction of drug-induced rotation and skilled forelimb use. Brain Res. Bull. 41:409-416; 1996.

6. Fricker, R. A.; Torres, E. M.; Hume, S. P.; Myers, R.; Opacka-Juffry, J.; Ashworth, S.; Dunnett, S. B. The effects of donor stage on the survival and function of embryonic striatal grafts. II. Correlation between positron emission tomography and reaching behaviour. Neuroscience 79:711-722; 1997.

7. Frim, D. M.; Uhler, T. A.; Short, M. P.; Ezzedine, Z. D.; Klagsbrun, M.; Breakefield, X. O.; Isacson, O. Effects of biologically delivered NGF, BDNF and bFGF on striatal excitotoxic lesions. Neuroreport 4:367-370; 1993.

8. Gerfen, C. R.; Staines, W. A.; Arbuthnott, G. W.; Fibiger, H. C. Crossed connections of the substantia nigra in the rat. J. Comp. Neurol. 207:283-303; 1982.

9. Herrera-Marschitz, M.; Meana, J. J.; Hökfelt, T.; You, Z. B.; Morino, P.; Brodin, E.; Ungerstedt, U. Cholecystokinin is released from a crossed corticostriatal pathway. Neuroreport 3:905-908; 1992.

10. Ilinsky, I. A.; Kultas-Ilinsky, K.; Rosina, A.; Haddy, M. Quantitative evaluation of crossed and uncrossed projections from basal ganglia and cerebellum to the cat thalamus. Neuroscience 21:207-227; 1987.

11. Jeyasingham, R. A.; Baird, A.; Meldrum, A.; Dunnett, S. B. Differential effects of unilateral striatal and nigrostriatal lesions on grip strength, skilled paw reaching and drug-induced rotation in the rat. Brain Res. Bull. 55:541548; 2001.

12. Marston, H. M.; Faber, E. S. L.; Crawford, J. H.; Butcher, S. P.; Sharkey, J. Behavioural assessment of endothelin-1 induced middle cerebral artery occlusion in the rat. Neuroreport 6:1067-1071; 1995.

13. Martinez-Serrano, A.; Björklund, A. Protection of the neo- striatum against excitotoxic damage by neurotrophin-producing, genetically modified neural stem cells. J. Neurosci. 16:4604-4616; 1996.

14. Miklyaeva, E. I.; Castañeda, E.; Whishaw, I. Q. Skilled reaching deficits in unilateral dopamine-depleted rats: Impairments in movement and posture and compensatory adjustments. J. Neurosci. 14:7148-7158; 1994.

15. Montoya, C. P.; Astell, S.; Dunnett, S. B. Effects of nigral and striatal grafts on skilled forelimb use in the rat. Prog. Brain Res. 82:459-466; 1990.

16. Montoya, C. P.; Campbell-Hope, L. J.; Pemberton, K. D.; Dunnett, S. B. The staircase test: A measure of independent forelimb reaching and grasping abilities in rats. J. Neurosci. Methods 36:219-228; 1991.

17. Nakao, N.; Brundin, P. Effects of alpha-phenyl-tert-butyl nitrone on neuronal survival and motor function following intrastriatal injections of quinolinate or 3-nitropropionic acid. Neuroscience 76:749-761; 1997.

18. Nakao, N.; Grasbon-Frodl, E. M.; Widner, H.; Brundin, P. DARPP-32-rich zones in grafts of lateral ganglionic eminence govern the extent of functional recovery in skilled paw reaching in an animal model of Huntington's disease. Neuroscience 74:959-970; 1996.

19. Pérez-Navarro, E.; Arenas, E.; Reiriz, J.; Calvo, N.; Alberch, J. Glial cell line derived neurotrophic factor protects striatal calbindin-immunoreactive neurons from excitotoxic damage. Neuroscience 75:345-352; 1996.

20. Pisa, M. Motor functions of the striatum in the rat: Critical role of the lateral region in tongue and forelimb reaching. Neuroscience 24:453-463; 1988.

21. Ungerstedt, U.; Arbuthnott, G. W. Quantitative recording of rotational behaviour in rats after 6-hydroxydopamine lesions of the nigrostriatal dopamine system. Brain Res. 24:485-493; 1970.

22. Watts, C.; Brasted, P. J.; Eagle, D. M.; Dunnett, S. B. Embryonic donor age and dissection influence striatal graft development and functional integration in a rodent model of Huntington's disease. Exp. Neurol. 163:85-97; 2000.

23. Whishaw, I. Q.; O'Connor, W. T.; Dunnett, S. B. The contributions of motor cortex, nigrostriatal dopamine and caudate-putamen to skilled forelimb use in the rat. Brain 109: 805-843; 1986.

24. Wilson, C. J. Morphology and synaptic connections of crossed corticostriatal neurons in the rat. J. Comp. Neurol. 263:567-580; 1987. 